\title{
SMALL-SCALE STRUCTURE IN YOUNG SUPERNOVA REMNANTS
}

\author{
Stephen P. Reynolds \\ Department of Physics, North Carolina State University \\ Raleigh, NC 27695-8202 USA
}

\begin{abstract}
Recent VLA observations of the shell supernova remnant SN $1006 \mathrm{AD}$ (Reynolds and Gilmore 1986) and the Crablike remnant 3C 58 (SN 1181 AD?; Reynolds and Aller 1987) show features at high resolution that contain information on details of particle acceleration and transport in the remnants. Thin arcs at the edge of SN 1006 require time-variable particle acceleration and/or magnetic field amplification. Filaments in 3C 58 probably result from interaction of pulsar-generated relativistic fluid with filaments of thermal gas formed early in the remnant's life by cooling or dynamical instabilities. Their sharp edges imply efficient scattering by Alfvén waves; as much as $1 \%$ of the large-scale magnetic energy density may be in magnetic turbulence on length scales of $10^{11} \mathrm{~cm}$.
\end{abstract}

Introduction. The wealth of information present in a high-resolution radio image of a supernova remnant can be translated into quantitative information about the behavior of the relativistic electrons and magnetic field responsible for the emission. The observation of structure on scales of order $0.1 \mathrm{pc}$ or less begins to constrain the diffusive properties of the post-shock turbulent medium. Very small-scale features place limits on the diffusion coefficient and effective mean free path of electrons; from this information, inferences can be drawn about the degree of disorder of the magnetic field. The origin of such features can pose significant problems for simple pictures of shock acceleration of electrons. In Crablike supernova remnants, the detailed nature of the nonthermal filamentation that dominates the appearance of 3C 58 and the Crab Nebula itself can provide clues to the nature and origin of the filaments; these questions bear on the evolutionary history of the remnant as well as on the characteristics of the turbulent plasma.

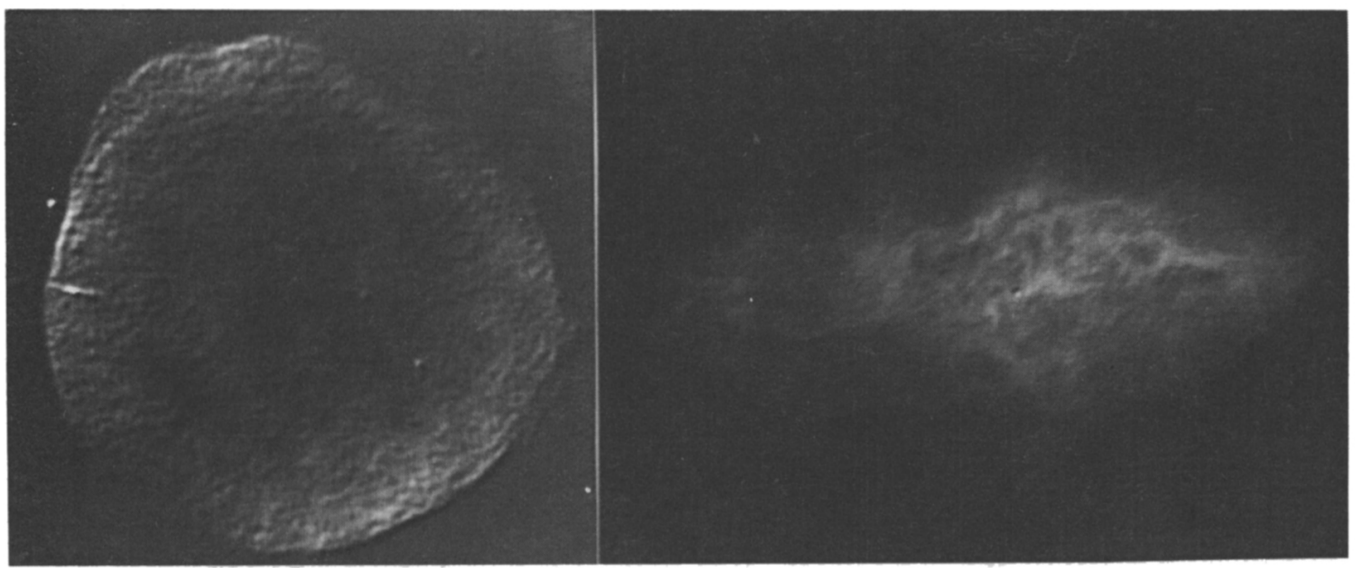

Fig. 1(a): $1.4 \mathrm{GHz}$ image of SN 1006.

Fig. 1(b): $1.4 \mathrm{GHz}$ image of 3C 58 . 
SN 1006. Figure $1(\mathrm{a})$ shows the $1.4 \mathrm{GHz}$ total-intensity image of SN 1006 (Reynolds and Gilmore 1986; RG) with a resolution of $16^{\prime \prime} \times 20^{\prime \prime}$. Contrast is set to enhance bright structure. The edge of the remnant is very sharp over much of its circumference; profiles show that it is unresolved. This suggests that the edge of the radio emission marks the blast wave, not the unstable contact discontinuity between shocked interstellar medium and supernova ejecta, since in the latter case one would expect a highly irregular appearance such as that of Cas $A$. However, the sharp edge in the eastern and southern parts of the remnant appears to be a narrow filament, dropping off as sharply behind (toward the remnant center) as in front. The eastern filament describes an arc of very nearly constant curvature and can be traced over almost $90^{\circ}$. The southern edge appears to show two parallel linear structures, again unresolved in the radial direction. These narrow filaments raise significant difficulties in modeling.

I assume a distance of $1.7 \mathrm{kpc}$ to SN 1006, a recent upward revision (Kirshner, Winkler, and Chevalier 1987). Then $20^{\prime \prime}$ corresponds to $0.17 \mathrm{pc}$. The profiles of RG indicate that the first minimum behind the eastern and southern filaments is at a level of about $70 \%$ of peak. This decrease over less than two beams or 0.3 pc cannot result from any smooth, steady, spherically symmetric process unless unacceptably huge gradients are invoked. To see this, assume that SN 1006 is in the adiabatic or Sedov phase of evolution, as indicated by the proper motion of the optical filament (Hesser and van den Bergh 1981). Then a parcel of gas currently at a relative position $\rho=r / r_{\text {shock }}$ was shocked at a time and radius calculable from the Sedov relations (Sedov 1959). The observed filament width of less than $40^{\prime \prime}$ out of a total radius of $15^{\prime}$ provides a conservative lower limit to the value of $\rho$ beyond which the emissivity must be larger, since any curvature would increase the projected width of this region. For $\rho=\mathbf{0 . 9 6}$, the Sedov relations imply that the material was shocked some 300 yr ago; for $\rho=0.98$, the material was shocked less than $170 \mathrm{yr}$ ago. Thus if the particle acceleration and magnetic field amplification/compression efficiencies have been constant over more than the last $300 \mathrm{yr}$, a wider feature than observed would be produced.

A quantitative estimate of the required gradients can be made assuming that very near the edge at least, the intensity varies as a power of radius. Then the rise in intensity $I_{\nu}$ by 1.4 over a fractional increase in projected radius $\rho$ of $4 \%$ or less requires that $I_{\nu}$ rise as at least $\rho^{8}$. Of course, this is completely inconsistent with the average profile of emission dropping reasonably toward the center; a crude average from one of the RG profiles suggests an increase more slow than $\rho^{3}$. Since the lines of sight corresponding to the filament peak and the adjacent interior minimum are so close together, the paths through the remnant are not greatly different, and the ratio in $I_{\nu}$ is roughly a ratio in mean emissivities. Thus the increase of a factor of about 1.4 would require an increase of the electron energy density by about the same factor, or an increase in the magnetic field strength by a factor 1.25 (since $j_{\nu} \propto B^{1-\alpha}$ and $\alpha \cong-0.6$ for SN 1006; Milne 1971). Now this must happen over a radial interval of less than $0.3 \mathrm{pc}$, but coherently over almost a quarter of the remnant circumference. The hint of two parallel filaments along the southern edge suggests that whatever might create this "burst" of emissivity might act repeatedly.

Another possibility exists for altering the synchrotron emissivity: the orientation of the mean magnetic field may change systematically with location. The simplest example is an exactly radial field. In order to investigate this effect quantitatively, I calculated theoretical profiles for such a geometry under several assumptions about the radius dependence of the magnetic field strength and relativistic particle density. If the latter quantities are roughly constant, the projection of the radial field produces a profile that 
peaks at $71 \%$ of the total radius, with a relative width at $70 \%$ of peak of $55 \%$ of the total radius. Adding a steep power-law increase to the magnetic field narrows the peak and moves it outwards, but even with the field growing roughly as $\rho^{4}$, the width at $70 \%$ of peak is still $23 \%$. This kind of simple geometry cannot account for the filaments; only a perturbation extensive in the tangential direction but very narrow in the radial, changing the field orientation by several tens of degrees, could produce the observed structures.

RG proposed that the filaments represented sheets with properties like those of the band across the remnant interior, but seen edge-on. While this is possible, a simple restriction of emissivity to a sector (an "orange slice" roughly perpendicular to the line of sight) cannot produce a narrow enough feature. Profiles of a constant-emissivity, radial field model so restricted have $70 \%$ widths of $21 \%$ even for a sector with opening angle only $20^{\circ}$. The emissivity must therefore decrease radially behind the edge, at a rate too great to have been constant over the remnant lifetime. The particle acceleration or magnetic-field enhancement rate must vary sharply with time.

Further information on the nature of the turbulent medium itself may be derived from the observation that the features remain narrow, implying that the diffusion of radio-emitting electrons is not rapid. Assume that the emissivity increase that produces the edge filaments was instantaneous. Then the observed limit on the filament widths is a limit on the diffusion coefficient for relativistic electrons. A one-dimensional delta-function of particles in a medium of diffusion coefficient $D=v \lambda / 3$, where $\lambda$ is the mean free path and $v$ the particle velocity, will diffuse in time $t$ into a Gaussian profile with dispersion $\sigma=(2 D t)^{1 / 2}$. Assuming that the observed width $\ell$ of the SN 1006 filaments is entirely due to electron diffusion from a plane of zero thickness, one obtains $\lambda<(3 / 2)\left(\ell^{2} / c t\right)$ or $\lambda<1.3 \times 10^{16}(\ell / 0.3 \mathrm{pc})^{2}(t / 100 \mathrm{yr})^{-1} \mathrm{~cm}$. While not yet a very strong limit, this length scale is already smaller than that on which the magnetic field can be completely tangled, or no significant polarization at all would be observed. If this scattering is due to resonant Alfvén waves with energy density $R$ times that in the large-scale magnetic field, the mean free path is roughly $(2 / \pi) r_{L} R^{-1}$ where $r_{L}$ is the electron Larmor radius (Wentzel 1974). For radio-emitting electrons in SN 1006, $r_{L} \sim 10^{11} \mathrm{~cm}$ in the equipartition magnetic field of $3 \times 10^{-5}$ Gauss, implying that at least $10^{-5}$ of the large-scale magnetic field energy is in magnetic turbulence on length scales of about $10^{11} \mathrm{~cm}$. Higher-resolution observations could substantially improve this limit.

3C 58. The filamentary structure in $3 \mathrm{C} 58$ must represent a totally different physical phenomenon. The bright filaments occur predominantly in the remnant interior. The relativistic particles and magnetic field responsible for the synchrotron emission presumably come from a central pulsar according to the conventional assumption for Crablike supernova remnants. While thermal material probably dominates the energy density in shell supernova remnants, the amount of thermal material in $3 \mathrm{C} 58$ is unknown, and possibly quite small, as judged from the faintness of optical features (van den Bergh 1978).

The VLA image of Figure 1(b) (Reynolds and Aller 1987: $2^{\prime \prime}$ resolution at $1.4 \mathrm{GHz}$ ), emphasizes the bright filamentary skeleton; the faint envelope is less apparent at this contrast. A few conspicuously long, narrow filaments can be seen, but most are fairly broad (widths $10^{\prime \prime}-30^{\prime \prime}$ ). However, the edges of filaments are sometimes unresolved and frequently narrower than $10^{\prime \prime}$ from valley to peak. Filament contrasts are comparable to those for the edge filaments of SN 1006; factors of 1.5 to 2 are typical for the ratio of a peak to the adjacent valley. If filaments are cylindrical and in the plane of the sky, this implies an emissivity increase of a factor of 30 to 40 in a typical filament. 
While the question of the origin of filaments in SN 1006 drew on the physics of shock acceleration, the origin of $3 \mathrm{C} 58$ 's filaments must be looked for elsewhere. Spectralindex maps between 1.4 and $4.9 \mathrm{GHz}$ (Reynolds and Aller 1987) show that there is no appreciable difference between the spectral index of bright filaments and that of the remnant mean. Thus filaments are neither locally shock-accelerated particles, nor compressed ambient cosmic-ray electrons; both sources would imply spectral indices $\alpha \lesssim-0.4\left(S_{\nu} \propto \nu^{\alpha}\right)$. The time-scales on which the pulsar-generated relativistic fluid could penetrate the surrounding thermal supernova ejecta are enormously long, so the thermal and relativistic gases are not coextensive. Cooling or dynamical instabilities acting on the thermal gas are the most likely mechanism to produce thermal filaments, but such mechanisms must act early while the shock driven by the injected relativistic fluid is slow and post-shock cooling times are short (Reynolds 1987). The nonthermal filaments then result from pulsar-injected material interacting with the thermal obstructions, creating nonthermal sheaths. The difficulty with this picture remains the faintness of optical emission, though the thermal gas required for this nucleation picture (roughly $0.2 M_{\odot}$, a tenth of that in the Crab Nebula) might, with some extinction, be difficult to detect.

Independently of the origin question, as with SN 1006 the observed sharpness of features constrains particle diffusion in 3C 58. For a distance to $3 \mathrm{C} 58$ of $2.6 \mathrm{kpc}$ (Green and Gull 1982), $2^{\prime \prime}$ corresponds to $8 \times 10^{16} \mathrm{~cm}$. The relations applied to $S N 1006$ above then give a limit on the mean free path of $\lambda<1.2 \times 10^{13}\left(\ell / 8 \times 10^{16} \mathrm{~cm}\right)^{2}(t / 806 \mathrm{yr})^{-1} \mathrm{~cm}$ in order that unresolved filament edges not be shallower than observed. For the equipartition field of $6 \times 10^{-5}$ Gauss, the Larmor radii of radio-emitting electrons are of order $10^{11} \mathrm{~cm}$; arguments like those presented above for SN 1006 imply that almost $1 \%$ of the large-scale magnetic field energy density is present in magnetic turbulence on the scale of $r_{L}$. This surprisingly strong result requires that the filaments be nearly as old as the remnant, as suggested above; if they were formed much later, or are transitory phenomena, the limit is weaker.

For both SN 1006 and 3C 58, radio polarimetry holds substantial promise for investigating the nature of particle diffusion. One should be able to learn about the degree of small-scale order in the field, and the geometry of large-scale order. Detailed theoretical modeling of profiles of features should further constrain the scattering lengths in the gas and, in SN 1006, can improve our understanding of shock acceleration.

\section{REFERENCES}

Green, D. A., and Gull, S. F. 1982, Nature, 299, 606.

Hesser, J. E., and van den Bergh, S. 1981, Ap. J., 251, 549.

Kirshner, R. P., Winkler, P. F., and Chevalier, R. A. 1987, Ap. J. (Letters), 315, L135.

Milne, D. K. 1971, Aust. J. Phys., 24, 757.

Reynolds, S. P. 1987, Ap. J., submitted.

Reynolds, S. P., and Aller, H. D. 1987, Ap. J., submitted.

Reynolds, S. P., and Gilmore, D. M. 1986, A.J., 92, 1138 (RG).

Sedov, L. I. 1959, Similarity and Dimensional Methods in Mechanics (New York: Academic), p. 219.

van den Bergh, S. 1978, Ap. J. (Letters), 220, L9.

Wentzel, D. G. 1974, Ann. Rev. Astr. Ap., 12, 71. 